$2016 / 31$

\title{
Who Benefits from Increased Competition Among Sellers on B2C Platforms?
}

Paul Belleflamme and ERIC Toulemonde

\section{YEARS OF}

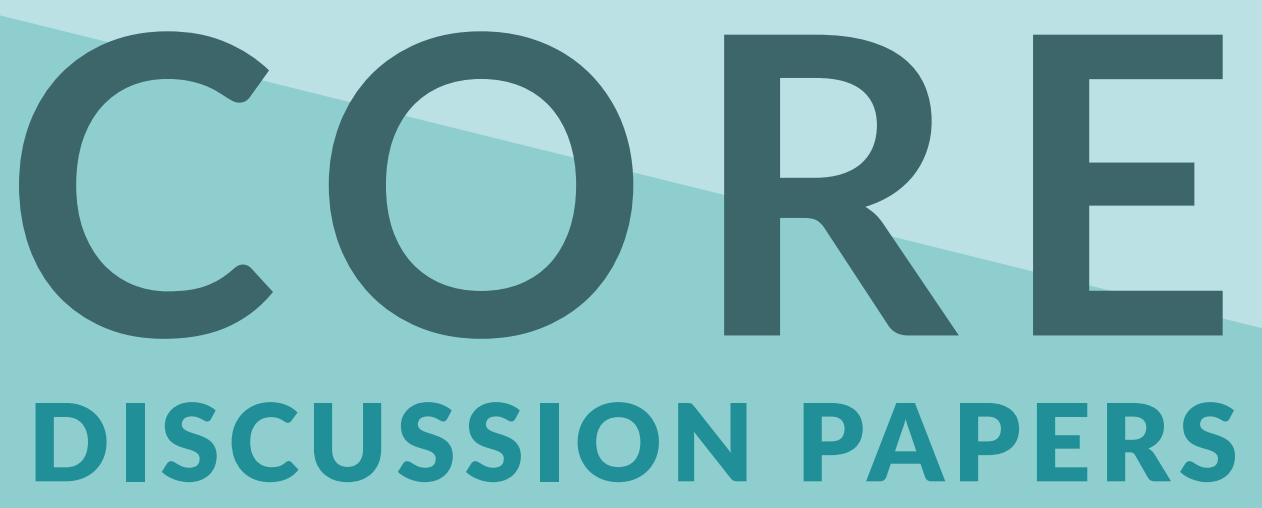




\section{CORE}

Voie du Roman Pays 34, L1.03.01

Tel (32 10) 474304

Fax (32 10) 474301

Email:immaq-library@uclouvain.be

http://www.uclouvain.be/en-44508.html 


\title{
Who benefits from increased competition among sellers on B2C platforms?*
}

\author{
Paul Belleflamme ${ }^{\dagger}$ \\ Eric Toulemonde $e^{\ddagger}$ \\ Université catholique de Louvain \\ University of Namur
}

August 26, 2016

\begin{abstract}
We introduce within-group external effects in the two-sided singlehoming model of Armstrong (2006). First, we propose a general characterization of the platform access fees at the symmetric equilibrium of the game. Second, we combine this general formulation with a specific modeling of the relationship between buyers and sellers on $\mathrm{B} 2 \mathrm{C}$ platforms, so as to analyze how changes in the underlying characteristics of the product market affect the equilibrium of the game. We show that sellers may be better off, and buyers worse off, in markets with more sellers. We also show that sellers and buyers prefer full product differentiation while platforms prefer no differentiation.

Keywords: Two-sided platforms, external effects, e-commerce

JEL-Classification: D43, L13, L86
\end{abstract}

\footnotetext{
${ }^{*}$ We thank Federico Etro for useful comments and suggestions on an earlier draft.

${ }^{\dagger} \mathrm{CORE}$ and Louvain School of Management, Université catholique de Louvain, 34 Voie du Roman Pays, B-1348 Louvain la Neuve, Belgium, Paul.Belleflamme@uclouvain.be; also affiliated with CESifo.

${ }^{\ddagger}$ Department of Economics and CERPE, University of Namur, 8 Rempart de la Vierge, B-5000 Namur, Belgium, Eric.Toulemonde@unamur.be.
} 


\section{Introduction}

In many economic situations, distinct groups of economic agents would benefit from interacting with one another but often fail to organize this interaction by their own forces because of high transaction costs or free-riding problems. There exists then a business opportunity for so-called multi-sided platforms (MSPs) to intermediate between the groups, so as to facilitate the interaction. By substantially bringing down transaction costs, the Internet and digital technologies have been a catalyst for the development of MSPs in a large variety of settings. Besides transaction systems (which provide a method for payment to buyers and sellers that are willing to use it; e.g., Visa, PayPal), exchanges (which help 'buyers' and 'sellers' search for feasible contracts and for the best prices; e.g., eBay, Booking.com), hardware 83 software systems (which allow applications developers and end users to interact; e.g., Mac OS, PlayStation), or matchmakers (which help members of one group to find the right 'match' within another group; e.g., Monster, Meetic), recent years have seen the emergence of peer-to-peer marketplaces (which facilitate the exchange of goods and services between 'peers'; e.g., Airbnb, Uber) and crowdfunding platforms (which allow entrepreneurs to raise funds from a 'crowd' of investors; e.g., Kickstarter, LendingClub).

The main function of MSPs is to internalize the external effects that the interaction between the various groups generate. Of primary interest are cross-group external effects, which make the well-being of the members of one group depend on the participation of the members of another group. In most of the examples given above, the cross-group effects are positive as each group benefits from a larger participation of other groups. Think, e.g., of merchants and clients for the Visa card, of game developers and gamers for the PlayStation, or of hosts and guests for Airbnb; in each example, the platform becomes more attractive for one group as it is more largely used by the other group. Negative cross-group effects are mainly observed on adfinanced media platforms; typically, as advertising is seen as a nuisance, a larger participation of the group of advertisers negatively affects the utility that the media platform brings to its end-users.

It is natural to focus on cross-group effects as they directly stem from the desire of the various groups to interact and, thereby, give their raison d'être to MSPs. However, when deciding on their strategies, MSPs must also factor in the potential existence of within-group external effects. These 
effects describe the fact that the attractiveness of a platform for the members of one group depends on the participation of the members of the very same group. Within-group effects are negative when the members of one group compete with one another to interact with the other group or because of some form of congestion. ${ }^{1}$ In contrast, there also exist sources of positive within-group effects. ${ }^{2}$

Most of the literature analyzing the competition between MSPs-following the seminal contributions of Caillaud and Jullien (2003), Rochet and Tirole (2003 and 2006), and Armstrong (2006)-has focused on cross-group effects, ignoring within-group effects. Notable exceptions are the following. Ellison and Fudenberg (2003) and Ellison, Fudenberg and Möbius (2004) show that negative within-side effects may contribute to the coexistence of competing two-sided platforms. In a similar vein, Belleflamme and Toulemonde (2009) examine the extent to which negative within-group effects among sellers may help a new platform operator lure buyers and sellers away from an existing free platform. Hagiu (2009) also introduces competition among sellers on a two-sided platform; competition stems from consumers' variable preference for variety over sellers' products, which turns out to be a key factor determining the optimal pricing structure either of a monopoly platform or of competing platforms. Belleflamme and Peitz (2010) extend their main setting by introducing congestion externalities among sellers, which proxy competition effects. Bardey, Cremer and Lozachmeur (2014) consider platforms that provide all their users with a larger utility when they increase their seller/buyer ratio (as is often the case in the education and health sectors); in this setting, there exist positive (resp. negative) withingroup effects among sellers (resp. buyers). More recently, Karle, Peitz and

\footnotetext{
${ }^{1}$ An example of competition is the one that exists among Uber drivers, who face a given set of passengers at any location and any point in time. Slee (2016) gives an example of congestion; talking about Airbnb visitors, he reports that "as their numbers grow, they erode the very atmosphere in which they bask and threaten the livability of the city for residents."

${ }^{2}$ One example is the presence of network effects among gamers who enjoy playing online game with a large crowd of potential opponents. Similarly, on MOOC (Massive Online Open Courses) platforms, a larger number of students for a particular course generates peer effects that contribute to improve course completion and performance (see Belleflamme and Jacqmin (2016) for a discussion). Another example, described in Belleflamme, Omrani and Peitz (2015), is the fact that a larger 'crowd' of funders on a crowdfunding platform increases the probability that any project will be realized, which benefits all funders.
} 
Reisinger (2015) examine how the degree of competition among sellers affect the possibility for non-differentiated platforms offering listing services to coexist at equilibrium. ${ }^{3}$

With this paper, we contribute to the literature in two main ways. First, we extend the model of Armstrong (2006) with two-sided singlehoming model to allow for any type of external effects. That is, in addition to the usual cross-group effects, the model also includes (positive or negative) within-group effects. Using this extended model, we come up with a very simple characterization of the platforms' access fees, the platforms' profits and the users' net surpluses at the symmetric equilibrium of the price competition game that the two platforms play. In particular, we establish that the equilibrium fee for buyers (resp. sellers) is the traditional Hotelling price (marginal cost + transportation cost) adjusted downward by the sum of the within- and cross-group external effects exerted by the switch of one buyer (resp. seller) from one platform to the other (starting from an equal division of buyers and sellers between the two platforms). It follows that the presence of positive (resp. negative) external effects from group $A$ to group $B$, or within group $A$, leads platforms to lower (resp. raise) the membership fee for group $A$ below (resp. above) the level that would prevail absent any external effect.

The second main contribution of the paper is to add (as Hagiu (2009) does) a microfoundation of the interaction between the agents of the two groups and, thereby, to endogenize the cross-group and within-group external effects. We focus on B2C platforms where buyers are final consumers of varieties of a differentiated good, which are produced by a set of sellers that compete à la Cournot. In this setting, cross-group effects are positive (buyers enjoy the presence of more sellers and vice versa), and within-group effects are negative for sellers (because of competition) and non-existent for buyers. Interestingly, these external effects cannot be additively separated,

\footnotetext{
${ }^{3}$ Other papers also consider competition among sellers but in markets where a single platform is active: Nocke, Peitz and Stahl (2007) study the link between the platform ownership and its prices; Galeotti and Moraga-González (2009) focus on a platform allowing consumers to search among competing sellers of differentiated products; in Lin, Li and Whinston (2011), sellers may decide to produce vertically differentiated products and the platform is shown to charge access fees to buyers that mitigates price competition among sellers; Tavalei and Santalo (2015) analyze, both theoretically and empirically, the effects of exogenous changes in competition within one group on the platform's optimal pricing of both groups.
} 
which contrasts with the simplifying assumption that is usually made in the literature. This microfoundation of external effects allows us to assess how platforms set their optimal fees according to characteristics of the market (namely, the degree of horizontal product differentiation, and the numbers of buyers and sellers).

Two important results emerge. First, when competing platforms intermediate trade, a seller may achieve a larger net surplus and a buyer, a lower net surplus in markets that count a larger number of sellers. This result stands in sharp contrast with what is observed when trade is not intermediated and is explained as follows: in markets with more sellers, platforms tend to charge lower fees to sellers, which outweighs the lower profits that sellers make on each transaction because of stronger competition among them; as for buyers, they benefit from stronger competition on the market but are charged higher fees by the platforms, with the possibility that the second effect dominates. The second result is that, at the equilibrium of the game, platforms and agents disagree about the preferred degree of product differentiation: platforms would prefer homogeneous products, while buyers and sellers would prefer perfectly differentiated products.

The rest of the paper is organized as follows. In Section 2, we lay out the general model of two-sided singlehoming with both cross- and within-group external effects. In Section 3, we use Cournot competition over horizontally differentiated products to generate buyers' surpluses and sellers' profits that exhibit intertwined external effects; we then perform comparative statics exercises to assess how stronger competition among sellers affects the equilibrium of the platform competition game. We conclude in Section 4.

\section{A general model of two-sided singlehoming}

We provide an abstract model of trade on a platform that closely follows the literature on two-sided markets and here, in particular, the setting of Armstrong (2006) and its extension by Belleflamme and Peitz (2010). This setting postulates that users on both sides-buyers and sellers-are heterogeneous with respect to their opportunity cost of joining a platform and supposes that platforms set membership fees. ${ }^{4}$ In particular, buyers and

\footnotetext{
${ }^{4}$ Rochet and Tirole (2003) consider heterogeneity in usage costs and consider the setting of usage fees. Hagiu (2006) considers sequential participation decisions by the two sides of the platform. Weyl (2010) and Weyl and White (2016) consider general non-linear
} 
sellers can interact on two platforms, 1 and 2, which are assumed to be located at the extreme points of the unit interval. Each group 'singlehomes' in the sense that a user can only be active on a single platform at a time; in our static framework, this means that before interaction takes place, users have to choose among the two platforms.

We suppose that there is an exogenous number of sellers $\left(N_{s} \geq 2\right)$ and an exogenous number of buyers $\left(N_{b} \geq 2\right)$. Like Belleflamme and Peitz (2010), we assume that before choosing which platform to visit, buyers and sellers draw independently their location from a uniform distribution on the unit interval. This location conditions their opportunity cost of visiting either platform as this cost increases linearly with the distance that separates the agent from the platform, at rates $\tau_{b}$ for buyers and $\tau_{s}$ for sellers. It is further assumed that each seller and each buyer has private information about his or her location.

We study the following four-stage game. At stage 1, platforms simultaneously set membership fees $M_{s}^{i}, M_{b}^{i}$ on the two sides of the market. At stage 2, sellers and buyers decide which platform to visit. At stage 3, sellers set the price or the quantity of their goods simultaneously. Finally, at stage 4 , buyers decide how much to purchase of the goods that are offered on the platform they have chosen to visit.

In this section, we focus on the first two stages of the game and postulate generic functions for the equilibrium payoffs of buyers and sellers resulting from stages 3 and 4 . In the next section, we introduce specific microfoundations of buyer-seller relationships so as to analyze the impact of the underlying market characteristics on the equilibrium of the game.

For now, we simply define buyer and seller surplus gross of any opportunity cost of visiting a platform as

$$
\begin{aligned}
& v_{b}^{i}=R_{b}+u\left(n_{b}^{i}, n_{s}^{i}\right)-M_{b}^{i}, \\
& v_{s}^{i}=R_{s}+\pi\left(n_{b}^{i}, n_{s}^{i}\right)-M_{s}^{i},
\end{aligned}
$$

where $M_{b}^{i}$ and $M_{s}^{i}$ are the membership fees set by intermediary $i$, and $R_{b}$ and $R_{s}$ are the stand-alone benefits that buyers and sellers derive from visiting any platform. As for $n_{b}^{i}$ (resp. $n_{s}^{i}$ ), it is the expected number of

pricing, so-called insulating tariffs. Armstrong and Wright (2007) consider the importance of multi-homing. Reisinger (2014) considers competition between two-sided platforms that use two-part tariffs. 
buyers (resp. sellers) that decide to interact on platform $i$; it is indeed an 'expected' number as buyers (resp. sellers) know only the distribution of the sellers' (resp. buyers') locations.

The functions $u\left(n_{b}^{i}, n_{s}^{i}\right)$ and $\pi\left(n_{b}^{i}, n_{s}^{i}\right)$ are the net gains from trade for, respectively, any buyer and any seller on platform $i$. They both potentially depend on the number of buyers and on the number of sellers who are present on the platform; both functions are supposed to be twice continuously differentiable in these two arguments. At this stage, we do not make any specific assumption regarding the signs of the derivatives of these two functions. For now, the model therefore encompasses any combination of within- and cross-group external effects.

Using expressions (1) and (2) as the equilibrium payoffs resulting from the market interaction that takes place in stages 3 and 4 , we solve for the subgame-perfect equilibrium in stages 1 and 2 of the game. We start with the buyers' and seller's platform choices; we analyze next the platforms' choice of membership fees.

Buyers' and sellers' platform choices. Let $b_{12}$ and $s_{12}$ denote, respectively, the location of the buyer and the seller who are indifferent between visiting platform 1 and visiting platform 2. Using expressions (1) and (2), along with our definition of the opportunity cost of visiting a platform, we find:

$$
\begin{aligned}
& v_{b}^{1}-\tau_{b} b_{12}=v_{b}^{2}-\tau_{b}\left(1-b_{12}\right) \Leftrightarrow b_{12}=\frac{1}{2}+\frac{v_{b}^{1}-v_{b}^{2}}{2 \tau_{b}}, \\
& v_{s}^{1}-\tau_{s} s_{12}=v_{s}^{2}-\tau_{s}\left(1-s_{12}\right) \Leftrightarrow s_{12}=\frac{1}{2}+\frac{v_{s}^{1}-v_{s}^{2}}{2 \tau_{s}} .
\end{aligned}
$$

In what follows, we assume that the stand-alone benefits $R_{b}$ and $R_{s}$ are sufficiently large so that all buyers and sellers participate in the market. Each side of the market is then divided into two groups at stage 2: buyers (resp. sellers) located between 0 and $b_{12}$ (resp. $s_{12}$ ) visit platform 1 and those located between $b_{12}$ (resp. $s_{12}$ ) and 1 visit platform 2 . It follows that $n_{b}^{1}=b_{12} N_{b}, n_{b}^{2}=\left(1-b_{12}\right) N_{b}, n_{s}^{1}=s_{12} N_{s}$, and $n_{s}^{2}=\left(1-s_{12}\right) N_{s}$, which correspond to the standard Hotelling specifications,

$$
\frac{n_{b}^{i}}{N_{b}}=\frac{1}{2}+\frac{v_{b}^{i}-v_{b}^{j}}{2 \tau_{b}}, \frac{n_{s}^{i}}{N_{s}}=\frac{1}{2}+\frac{v_{s}^{i}-v_{s}^{j}}{2 \tau_{s}}
$$

Using the expressions for buyer and seller surplus (1) and (2), and the facts that $n_{b}^{j}=N_{b}-n_{b}^{i}$ and $n_{s}^{j}=N_{s}-n_{s}^{i}$, we obtain the following expressions 
for the numbers of buyers and sellers at the two platforms:

$$
\left\{\begin{array}{l}
\frac{n_{b}^{i}}{N_{b}}=\frac{1}{2}+\frac{1}{2 \tau_{b}} \Delta^{u}\left(n_{b}^{i}, n_{s}^{i}\right)-\frac{1}{2 \tau_{b}}\left(M_{b}^{i}-M_{b}^{j}\right), \\
\frac{n_{s}^{i}}{N_{s}}=\frac{1}{2}+\frac{1}{2 \tau_{s}} \Delta^{\pi}\left(n_{b}^{i}, n_{s}^{i}\right)-\frac{1}{2 \tau_{s}}\left(M_{s}^{i}-M_{s}^{j}\right),
\end{array}\right.
$$

where

$$
\begin{aligned}
\Delta^{u}\left(n_{b}^{i}, n_{s}^{i}\right) & \equiv u\left(n_{b}^{i}, n_{s}^{i}\right)-u\left(N_{b}-n_{b}^{i}, N_{s}-n_{s}^{i}\right), \\
\Delta^{\pi}\left(n_{b}^{i}, n_{s}^{i}\right) & \equiv \pi\left(n_{b}^{i}, n_{s}^{i}\right)-\pi\left(N_{b}-n_{b}^{i}, N_{s}-n_{s}^{i}\right) .
\end{aligned}
$$

We also introduce the following notation:

$$
\begin{aligned}
\Delta_{b}^{u} & \equiv \frac{\partial \Delta^{u}\left(n_{b}^{i}, n_{s}^{i}\right)}{\partial n_{b}^{i}}, \Delta_{s}^{u} \equiv \frac{\partial \Delta^{u}\left(n_{b}^{i}, n_{s}^{i}\right)}{\partial n_{s}^{i}} \\
\Delta_{b}^{\pi} & \equiv \frac{\partial \Delta^{\pi}\left(n_{b}^{i}, n_{s}^{i}\right)}{\partial n_{b}^{i}}, \Delta_{s}^{\pi} \equiv \frac{\partial \Delta^{\pi}\left(n_{b}^{i}, n_{s}^{i}\right)}{\partial n_{s}^{i}} .
\end{aligned}
$$

In words, the function $\Delta^{u}\left(n_{b}^{i}, n_{s}^{i}\right)$ measures the differential in buyers' net gains from trade between platforms $i$ and $j$ when there are $n_{b}^{i}$ buyers and $n_{s}^{i}$ sellers on platform $i$. The derivatives $\Delta_{b}^{u}$ and $\Delta_{s}^{u}$ measures the sensitivity of this differential to an infinitesimal increase in the number of, respectively, buyers or sellers on platform $i$; the function $\Delta^{\pi}\left(n_{b}^{i}, n_{s}^{i}\right)$ and derivatives $\Delta_{b}^{\pi}$ and $\Delta_{s}^{\pi}$ are defined accordingly for sellers.

The system of equations (3) implicitly determines the demand functions for platform $i, n_{b}^{i}\left(M_{b}^{i}, M_{s}^{i} ; M_{b}^{j}, M_{s}^{j}\right)$ and $n_{s}^{i}\left(M_{b}^{i}, M_{s}^{i} ; M_{b}^{j}, M_{s}^{j}\right)$, which depend on the combination of the four fees. ${ }^{5}$ Assuming that the intermediary's cost per buyer is $C_{b}$ and per seller is $C_{s}$, we can write platform $i$ 's maximization program as

$$
\max _{M_{b}^{i}, M_{s}^{i}} \Pi^{i}=\left(M_{b}^{i}-C_{b}\right) n_{b}^{i}(\cdot)+\left(M_{s}^{i}-C_{s}\right) n_{s}^{i}(\cdot) .
$$

Expressing platform $j$ 's program in a similar way and solving, we obtain a system of four first-order conditions whose solution leads to the following proposition (see Appendix 5.1 for the proof). ${ }^{6}$

\footnotetext{
${ }^{5}$ We assume that the functions $u$ and $\pi$ are such that the system (3) leads to a unique solution $\left(n_{b}^{i}, n_{s}^{i}\right) \in\left[0, N_{b}\right] \times\left[0, N_{s}\right]$, which is well-behaved in the sense that both $n_{b}^{i}$ and $n_{i}^{s}$ are decreasing functions of $\left(M_{b}^{i}-M_{b}^{j}\right)$ and $\left(M_{s}^{i}-M_{s}^{j}\right)$. We will come back to this assumption in Section 3 when introducing specific functional forms for $u$ and $\pi$.

${ }^{6}$ We assume that given the functions $u\left(n_{b}^{i}, n_{s}^{i}\right)$ and $\pi\left(n_{b}^{i}, n_{s}^{i}\right), \tau_{b}$ and $\tau_{s}$ are sufficiently large to ensure that the second-order conditions for profit maximization are satisfied.
} 
Proposition 1 At the symmetric subgame-perfect equilibrium, both platforms set the following membership fees, respectively to buyers and to sellers

$$
\begin{aligned}
& M_{b}^{*}=C_{b}+\tau_{b}-\frac{N_{b}}{2} \Delta_{b}^{u}-\frac{N_{s}}{2} \Delta_{b}^{\pi}, \\
& M_{s}^{*}=C_{s}+\tau_{s}-\frac{N_{b}}{2} \Delta_{s}^{u}-\frac{N_{s}}{2} \Delta_{s}^{\pi},
\end{aligned}
$$

and achieve the following profits

$$
\Pi^{*}=\frac{1}{2}\left(\tau_{b} N_{b}+\tau_{s} N_{s}\right)-\frac{1}{4}\left(\Delta_{b}^{u} N_{b}^{2}+\Delta_{s}^{\pi} N_{s}^{2}+\left(\Delta_{b}^{\pi}+\Delta_{s}^{u}\right) N_{b} N_{s}\right),
$$

where $\Delta_{b}^{u}, \Delta_{b}^{\pi}, \Delta_{s}^{u}$, and $\Delta_{s}^{\pi}$ are evaluated at $\left(n_{b}^{i}, n_{s}^{i}\right)=\left(\frac{1}{2} N_{b}, \frac{1}{2} N_{s}\right)$.

Proposition 1 shows that the equilibrium membership fee for buyers (resp. sellers) is the traditional Hotelling price (marginal cost + transportation cost) adjusted downward by the marginal within- and cross-group external effects exerted by the switch of a buyer (resp. seller) from one platform to the other (starting from an equal division of buyers and sellers between the two platforms), multiplied by the respective numbers of agents on which these effects are exerted.

We observe in expressions (4) and (5) that the platforms' equilibrium prices depend on the nature of the within- and cross-group external effects. In the complete absence of external effects within and across groups, prices would be as in the Hotelling model. The presence of positive (resp. negative) external effects from group $A$ to group $B$, leads platforms to lower (resp. raise) the membership fee for group $A$ below (resp. above) the level that would prevail absent any external effect. This is the standard result of Armstrong (2006). We add a new result related to the presence of external effects within groups. Positive (resp. negative) external effects within groups leads platforms to lower (resp. raise) the membership fee for the group below (resp. above) the level that would prevail absent any external effect. We also observe in expression (6) that the platforms' equilibrium profits decrease (resp. increase) when cross-group or within-group external effects become more positive (resp. more negative). Clearly, the exact nature of the various external effects depend on the modeling of the buyer-seller interaction, as we now examine.

\section{Buyer-seller relationships on the platform}

We now propose specific microfoundations for the interaction between buyers and sellers. We consider a B2C setting where buyers are final consumers of 
varieties of a differentiated good, which are produced by a set of sellers that compete à la Cournot. We start by deriving the equilibrium surplus for buyers and profits for sellers; next, we introduce these values within our previous analysis.

\subsection{Stages 3 and 4}

Suppose that each buyer has the following quadratic utility function:

$$
U\left(q_{0} ; q_{1}, q_{2}, \ldots, q_{n}\right)=a \sum_{s=1}^{n} q_{s}-\frac{1}{2}\left(\sum_{s=1}^{n} q_{s}^{2}+\gamma \sum_{s=1}^{n} \sum_{t \neq s} q_{s} q_{t}\right)+q_{0}
$$

where $q_{0}$ is the Hicksian composite commodity (with a price normalized to $1), n$ is the number of varieties of the differentiated good that the buyer has access to, and $0 \leq \gamma \leq 1$ measures the strength of the substitutability among varieties (varieties are independent for $\gamma=0$ and homogeneous for $\gamma=1)$. The buyer maximizes her utility $U\left(q_{0} ; q_{1}, q_{2}, \ldots, q_{n}\right)$ subject to the budget constraint $y=q_{0}+\sum_{s=1}^{n} p_{s} q_{s}$. This gives rise to the following inverse demand functions $p_{s}=a-q_{s}-\gamma Q_{-s}$ (with $Q_{-s}=\sum_{t \neq s} q_{t}$ ) for strictly positive prices and zero otherwise.

In the symmetric case where all firms set the same price $p$ (which is a characteristic of symmetric equilibrium), we have $Q(n, \gamma)=n(a-p) /(1+$ $\gamma(n-1))$, which is clearly an increasing function of the number of varieties $n$, and a decreasing function of the degree of substitutability $\gamma$; this is because buyers are assumed to love variety. ${ }^{7}$ It is important to keep these effects in mind when we perform comparative statics exercises.

To derive the functions $\pi(\cdot)$ and $u(\cdot)$, consider platform $i$ where $n_{b}^{i}$ buyers and $n_{s}^{i}$ sellers interact. Each of the $n_{b}^{i}$ buyers has the utility function given by (7). Each of the $n_{s}^{i}$ sellers produces one variety of the differentiated product at a constant marginal cost $c$; sellers compete à la Cournot.

Seller $s$ chooses its quantity $q_{s}$ to maximize $n_{b}^{i}\left(a-c-q_{s}-\gamma Q_{-s}\right) q_{s}$. The first-order condition yields $2 q_{s}=a-c-\gamma Q_{-s}$. Summing over the $n_{s}^{i}$ sellers and writing $Q$ for $\sum_{s=1}^{n_{s}^{i}} q_{s}$, one has $2 Q=n_{s}^{i}(a-c)-\gamma\left(n_{s}^{i}-1\right) Q$. Solving for $Q$, one obtains $Q=n_{s}^{i}(a-c) /\left(2+\gamma\left(n_{s}^{i}-1\right)\right)$. By symmetry,

\footnotetext{
${ }^{7}$ This specification of linear demand functions is often attributed to Singh and Vives (1984). Shubik and Levitan (1980) propose an alternative linear demand model where market size is kept fixed when the number of varieties increases. For a discussion of the microfoundations for linear demand product differentiation models, see Martin (2009).
} 
each seller produces the same quantity at the Cournot-Nash equilibrium: $q_{s}^{*}=Q / n_{s}^{i}$. It is easily computed that $p_{s}^{*}-c=q_{s}^{*}$. Setting, without loss of generality, $a-c=1$, we compute the equilibrium profit for each seller as

$$
\pi\left(n_{b}^{i}, n_{s}^{i}\right)=\frac{n_{b}^{i}}{\left(2+\gamma\left(n_{s}^{i}-1\right)\right)^{2}} .
$$

To compute a buyer's surplus, we substitute the inverse demand functions for $p_{s}\left(s=1, . ., n_{s}^{i}\right)$ into the budget constraint:

$$
C S\left(q_{0}, q_{1}, q_{2}, \ldots, q_{n_{s}^{i}}\right)=\frac{1}{2}\left(\sum_{s=1}^{n_{s}^{i}} q_{s}^{2}+\gamma \sum_{s=1}^{n_{s}^{i}} \sum_{t \neq s} q_{s} q_{t}\right) .
$$

Given that at the Cournot-Nash equilibrium, $q_{s}^{*}=Q / n_{s}^{i}$ for all $s$, one obtains

$$
u\left(n_{b}^{i}, n_{s}^{i}\right)=\frac{n_{s}^{i}\left(1+\gamma\left(n_{s}^{i}-1\right)\right)}{2\left(2+\gamma\left(n_{s}^{i}-1\right)\right)^{2}} .
$$

The functions (8) and (9) exhibit the following external effects. Regarding buyers, we see that the surplus is independent of the number of buyers: $u_{b}=0$; as for the influence of an additional seller, it can be checked that $u_{s}>0$ : an additional seller exerts a positive external effect on buyers. On the sellers' side, it is directly observed that $\pi(\cdot)$ increases with $n_{b}^{i}$ and decreases with $n_{s}^{i}$; that is, an additional buyer exerts positive external effects and an additional seller, negative external effects on sellers. ${ }^{8}$ As for the effects of product differentiation, we see that when it increases (i.e., when $\gamma$ decreases), the seller's profit increases (as competition is relaxed) and the buyer's surplus increases (because buyers value product variety). Note that at the symmetric equilibrium $\left(N_{b} / 2, N_{s} / 2\right)$, prices decrease with $\gamma$ and $N_{s}$ :

$$
p_{s}^{*}=c+\frac{2}{4+\gamma\left(N_{s}-2\right)} .
$$

We can now use expressions (8) and (9) to give an explicit form to the platforms' membership fees at the subgame-perfect equilibrium of the game in its three scenarios. ${ }^{9}$

\footnotetext{
${ }^{8}$ The within-group effect among sellers vanishes when $\gamma=0$, i.e., when sellers produce perfectly differentiated varieties.Then, there is no competition among sellers and we are back to the surplus functions used in Belleflamme and Peitz (2010), where $N_{b}$ and $N_{s}$ are set equal to unity: $u\left(n_{b}^{i}, n_{s}^{i}\right)=\tilde{u} n_{s}^{i}$ and $\pi\left(n_{b}^{i}, n_{s}^{i}\right)=\tilde{\pi} n_{b}^{i}$, with $\tilde{u} \equiv 1 / 8$ and $\tilde{\pi}=1 / 4$.

${ }^{9}$ Numerical simulations allow us to check that we obtain unique and interior values of $n_{i}^{b}$ and $n_{i}^{s}$, which reacts to changes in fees in the expected way. A full analytical verification is, however, impossible as solving system (3) leads to a polynomial of degree 9 in $n_{i}^{s}$.
} 


\section{$3.2 \quad$ Stages 1 and 2}

We now examine the platforms' pricing decisions. Using expressions (8) and (9), we compute the values of $\Delta_{b}^{u}, \Delta_{s}^{u}, \Delta_{b}^{\pi}, \Delta_{s}^{\pi}$ at $\left(N_{b} / 2, N_{s} / 2\right)$ as

$$
\begin{aligned}
\Delta_{b}^{u} & =0, \Delta_{s}^{u}=4 \frac{\gamma(3-\gamma) N_{s}+2(1-\gamma)(2-\gamma)}{\left(4+\gamma\left(N_{s}-2\right)\right)^{3}}, \\
\Delta_{b}^{\pi} & =\frac{8}{\left(4+\gamma\left(N_{s}-2\right)\right)^{2}}, \Delta_{s}^{\pi}=-\frac{16 \gamma N_{b}}{\left(4+\gamma\left(N_{s}-2\right)\right)^{3}} .
\end{aligned}
$$

We observe for future reference that these measures of the cross- and withingroup external effects are jointly affected by a change in the underlying conditions of the product market. In particular, we find that both $\Delta_{s}^{u}$ and $\Delta_{b}^{\pi}$ are positive and decrease with $\gamma$ and $N_{s}$, and are independent of $N_{b}$; on the other hand, $\Delta_{s}^{\pi}$ is negative and, in absolute value, it decreases in $N_{s}$, increases in $N_{b}$ and decreases in $\gamma$ as long as $N_{s}>2(1+\gamma) / \gamma$.

Plugging the values of $\Delta_{b}^{u}, \Delta_{s}^{u}, \Delta_{b}^{\pi}, \Delta_{s}^{\pi}$ into expression (4) and (5), we obtain the platforms' equilibrium membership fees:

$$
\begin{aligned}
M_{b}^{*} & =C_{b}+\tau_{b}-\frac{4 N_{s}}{\left(4+\gamma\left(N_{s}-2\right)\right)^{2}}, \\
M_{s}^{*} & =C_{s}+\tau_{s}+2 N_{b} \frac{\gamma(1+\gamma) N_{s}-2(1-\gamma)(2-\gamma)}{\left(4+\gamma\left(N_{s}-2\right)\right)^{3}} .
\end{aligned}
$$

We can now compute the equilibrium net surpluses for buyers and sellers as (where $V_{k} \equiv R_{k}-C_{k}-\tau_{k}, k=b, s$ )

$$
\begin{aligned}
& v_{b}^{*}=V_{b}+\frac{10+\gamma\left(N_{s}-2\right)}{2\left(4+\gamma\left(N_{s}-2\right)\right)^{2}} N_{s}, \\
& v_{s}^{*}=V_{s}-2 \frac{\gamma^{2} N_{s}-2(2-\gamma)^{2}}{\left(4+\gamma\left(N_{s}-2\right)\right)^{3}} N_{b} .
\end{aligned}
$$

As for the platforms' equilibrium profits, they are computed as

$$
\Pi^{*}=\frac{1}{2}\left(\tau_{b} N_{b}+\tau_{s} N_{s}\right)-\frac{\gamma(1-\gamma) N_{s}+2(2-\gamma)(3-\gamma)}{\left(4+\gamma\left(N_{s}-2\right)\right)^{3}} N_{b} N_{s} .
$$

\subsection{What if the product market is more competitive?}

We now want to analyze how the equilibrium of the game changes after a modification of the underlying conditions of the product market, namely the degree of product substitutability $(\gamma)$, the number of sellers $\left(N_{s}\right)$ and the number of buyers $\left(N_{b}\right)$. Note that to reach expressions (3) in the general 
model, we have assumed that all buyers and sellers participate in the market. Hence, the model is not suited for the analysis of endogenous changes in the number of agents such as those considered in models with free entry. Indeed, starting from a free entry equilibrium, any marginal increase in the sellers' fee pushes to the exit the marginal firm, meaning that the market would not be covered anymore, which contradicts the initial assumption of our model. Even though we cannot examine endogenous changes in the number of agents, we can still compare markets characterized by different number of sellers and we can show which agents gain or lose from different levels of competition in different markets.

An increase in $\gamma$, an increase in $N_{s}$ or a decrease in $N_{b}$ make the product market more competitive. What is interesting, when trade is mediated by platforms, is that each of these variables affects not only the surplus that the agents obtain when trading on the platform, but also the fees that they pay to access the platforms because these variables shape the cross-group and within-group external effects. Hence, their impact on net surpluses of buyers and sellers, as well as on the platforms' profits, is a priori not clear. In the rest of this section, we try to disentangle the various effects for each category of agents. To help the reader, Figures 1 and 2 show how fees and surpluses vary with competition. The top-right corner of the figures characterizes markets where competition is stronger, because either sellers are more numerous or the products they sell are less differentiated; the opposite goes for the bottom-left corner.

\subsubsection{Buyers}

A first immediate finding is that buyers are indifferent about their total number $\left(N_{b}\right)$ as it affects neither their surplus from trade nor their access fee to the platforms. Another rather straightforward finding is that buyers enjoy more product differentiation (i.e., a decrease in $\gamma$ ). There are two reasons for that. First, a lower $\gamma$ raises the surplus from trade $u\left(N_{s} / 2\right)$ (it has a direct positive effect on the buyer's utility function (7) that outweighs the negative effect of higher product prices resulting from milder competition). Second, platforms charge buyers a lower access fee as $\gamma$ decreases (this is because the cross-side external effects that buyers exert on sellers, $\Delta_{b}^{\pi}$, gets larger when $\gamma$ decreases).

In contrast, the effects of the number of sellers $\left(N_{s}\right)$ are mixed. When $N_{s}$ 


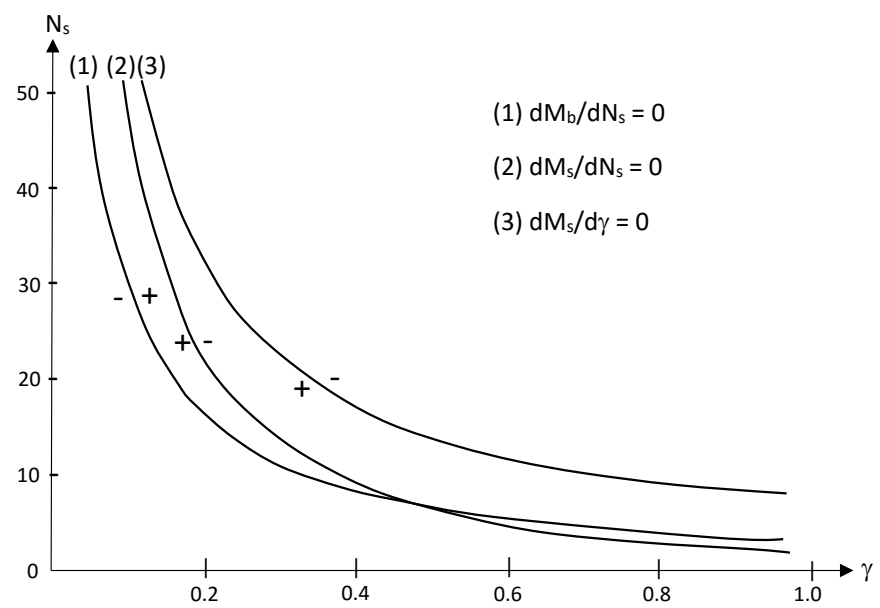

Figure 1: Effects of competition on equilibrium fees

increases, buyers enjoy a larger surplus from trade (because of more varieties and stronger competition) but they may have to pay a larger access fee to platforms (see Figure 1). To see the latter point, note that when $N_{s}$ increases, the cross-side external effects that buyers exert on sellers, $\Delta_{b}^{\pi}$, gets smaller but it is exerted on a larger number of them ( $N_{s} / 2$ increases). Putting everything together, we find that buyers enjoy the presence of more sellers if their number is not too large to start with; more precisely, $N_{s}$ must not be larger than some threshold, represented by (1) in Figure 2. We record our results in the following lemma.

Lemma 1 (i) An increase in product differentiation raises the buyers' equilibrium net surplus. (ii) A larger number of sellers has an ambiguous effect on the the buyers' equilibrium net surplus: positive if competition is weak, negative otherwise.

\subsubsection{Sellers}

The effects of stronger competition are clear for the sellers profits but ambiguous for their equilibrium fees. Computing the equilibrium sellers' profits, $\pi\left(N_{b} / 2, N_{s} / 2\right)$, from expression (8), we see that it decreases as $\gamma$ or $N_{s}$ increases, or as $N_{b}$ decreases.

As for the sellers' fee expressed in (11), two forces work in opposite directions: on the one hand, the positive cross-group effect $\left(\Delta_{s}^{u}>0\right)$ tends to 


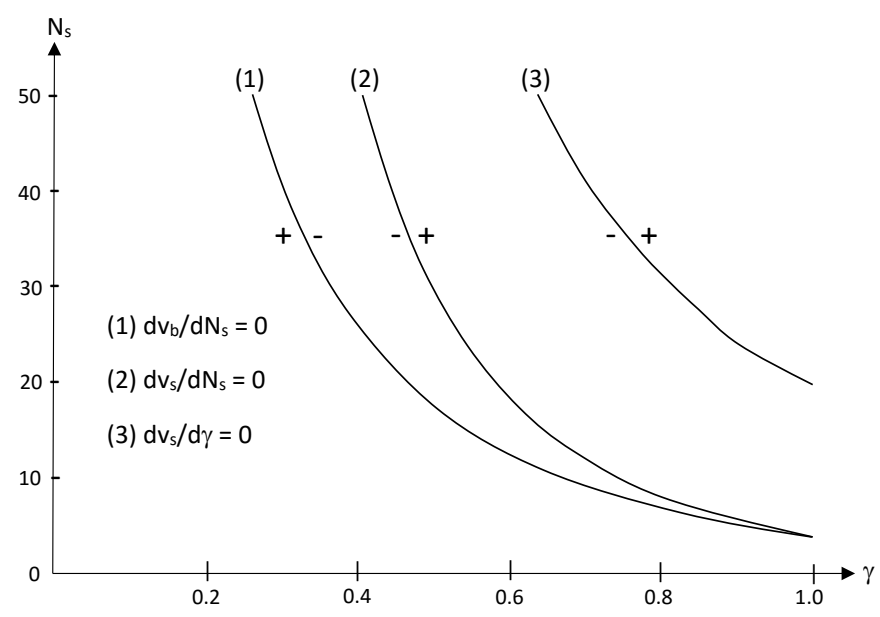

Figure 2: Effects of competition on equilibrium net surpluses

reduce the sellers' fee but on the other hand, the negative within-group effect $\left(\Delta_{s}^{\pi}<0\right)$ tends to increase it. Moreover, we have shown above that these two effects may respond differently to the three measures of the strength of competition and, sometimes, in non-monotonic ways. As a result, the signs of the derivatives $\partial M_{s}^{*} / \partial \gamma, \partial M_{s}^{*} / \partial N_{s}$ and $\partial M_{s}^{*} / \partial N_{b}$ cannot be easily ascertained. Figure 1 shows that sellers' fees decrease with competition when competition is already strong.

Putting everything together, for increased competition to benefit sellers, we would need that platforms reduce their equilibrium fee in such an extent that this compensates for the decrease in the sellers profits. As we see in Figure 2, this arises in markets where competition is already strong (large $N_{s}$ and $\gamma$ ). Finally, $\partial v_{s}^{*} / \partial N_{b}<0$ if $N_{s}>2(2-\gamma)^{2} / \gamma^{2}$ (e.g., for $\gamma=1 / 2$, we need $\left.N_{s} \geq 18\right)$. The next lemma summarizes these results.

Lemma 2 The sellers' equilibrium net surplus may well be larger in markets where competition is stronger.

Combining the results of Lemmata 1 and 2, we can establish the possibility of the following paradoxical situation.

Proposition 2 When trade is intermediated by competing platforms, sellers may prefer more competitive markets and buyers, less competitive markets. 
For this to happen, the increase in competition has to come from a larger number of sellers. ${ }^{10}$ Our previous analyses show that $\partial v_{b}^{*} / \partial N_{s}<0$ and $\partial v_{s}^{*} / \partial N_{s}>0$ are both satisfied provided that the couple $\left(N_{s}, \gamma\right)$ is located at the right of curve (2) in Figure 2. Here is an example that illustrates the result: take $\gamma=1 / 2$, meaning that we need $N_{s} \geq 30$. Let us then compare the net surpluses of buyers and sellers when going from a market with, say, $N_{s}=40$ to a market with one more seller, $N_{s}=41$ (taking $N_{b}=100$ ); as the following table shows, buyers prefer the market with fewer sellers, while the opposite prevails for sellers.

\begin{tabular}{|c|c|c|}
\hline & $v_{b}^{*}$ & $v_{s}^{*}$ \\
\hline$N_{s}=40$ & $V_{b}+1.0964$ & $V_{s}-0.0904$ \\
\hline$N_{s}=41$ & $V_{b}+1.0951$ & $V_{s}-0.0886$ \\
\hline
\end{tabular}

The intuition for this result is as follows. When trade is intermediated, the well-being of buyers and sellers also depends on the fees that platforms charge them to enable trade. Although the gains from trade unequivocally increase for buyers and decrease for sellers when the number of sellers on the market increases, the equilibrium fees set by the competing platforms may make the net surpluses of the agents move in the opposite direction. A larger number of sellers may indeed induce platforms to raise their fee on the buyer side and reduce it on the seller side.

\subsubsection{Platforms}

Regarding platforms, it makes little sense to analyze the effects of a larger number of sellers or of buyers, as platforms benefit directly from having (exogenously) more traders from which they can collect fees. ${ }^{11}$ We therefore focus on the effects of increased product substitutability. We have shown above that following an increase in $\gamma$, platforms manage to charge buyers

\footnotetext{
${ }^{10}$ If competition increases through a reduction of product differentiation (i.e., larger $\gamma$ ), both buyers and sellers are hurt when trade is not intermediated (recall that buyers love variety in our setting). When trade is intermediated, buyers have even more reasons to be hurt by an increase in $\gamma$ but sellers may benefit from it (because platforms have an incentive to set lower fees).

${ }^{11}$ Furthermore, this direct positive effect is likely to outweigh any negative indirect effect that could stem from a reduction in the equilibrium fees for sellers and/or buyers. This is because the direct effect is proportional to the values of $\tau_{s}$ and $\tau_{b}$, which are, by assumption, sufficiently large so as to guarantee the coexistence of platforms at equilibrium.
} 
a higher fee but it is not clear whether they can also do so for sellers. If both fees increase, then platforms profits necessarily increase as well; but if higher values of $\gamma$ lead to a lower seller fee, then opposite forces are exerted on the platforms' profits, which are mitigated by the respective numbers of buyers and sellers. It turns out, however, that platforms profits always increase when products become less differentiated. We record therefore the following lemma (which we prove in Appendix 5.3).

Lemma 3 For a given number of sellers and buyers, platforms achieve larger equilibrium profits when sellers offer less differentiated products.

The intuition behind this result is the following. We have already explained above that a decrease in product differentiation allows platforms to charge higher fees to buyers. This is because the stronger competition among sellers drives them to choose a platform not so much for the high number of buyers, but rather for the small number of sellers, that this platform may attract. As a result, the two platforms compete less fiercely to attract buyers as they become less instrumental to attract additional sellers. Following the same reasoning, there is also ground for platforms to raise the sellers fee as product differentiation decreases. However, another force works in the opposite direction: as products become closer substitutes, sellers make lower profits, which decreases their willingness to pay to access platforms. As we noted above, the balance between these two forces is ambiguous: when $\gamma$ increases, the equilibrium seller fee may increase or decrease. In the former case, platforms profits increase both on the buyer and on the seller side as competition among sellers increases. In the latter case, platforms profits increase on the buyer side but decrease on the seller side; however, Lemma 3 shows that the net effect is always positive.

We conclude this section by collecting the previous results and showing that agents have diverging preferences regarding the degree of product differentiation that should prevail on the market. Unambiguously, buyers are better off when product differentiation is the largest $(\gamma=0)$ and platforms when it is the lowest $(\gamma=1)$. As for sellers, their net surplus is convex in $\gamma$ and it is easily shown that it is larger at $\gamma=0$ than at $\gamma=1 .^{12}$ The next proposition records the results.

\footnotetext{
${ }^{12}$ The sellers' net surplus reaches a minimum at $\gamma=\gamma_{s} \equiv\left(2 \sqrt{6 N_{s}}-4\right) /\left(N_{s}-2\right)$, with $\gamma_{s}>1$ for $N_{s}<20$. Tt $\gamma=0, v_{s}^{*}=V_{s}+N_{b} / 4$; at $\gamma=1, v_{s}^{*}=V_{s}-2 N_{b}\left(N_{s}-2\right) /\left(N_{s}+2\right)^{3}$.
} 
Proposition 3 At the symmetric equilibrium of the platform pricing game, both sellers and buyers are better off when varieties are perfectly differentiated. However, the opposite prevails for platforms, which achieve larger profits when sellers produce homogeneous varieties.

\section{Conclusion}

In this paper, we have explicitly included within-group external effects in the two-sided singlehoming model of Armstrong (2006), which has become the workhorse model to analyze price competition between two horizontally differentiated two-sided platforms. We have first proposed a general - and surprisingly simple - characterization of the platform access fees at the symmetric equilibrium of the game. We have then coupled this general formulation with a specific modeling of the relationship between buyers and sellers on a B2C platforms. Doing so, we have shown that cross- and within-group external effects are intertwined and jointly influenced by the underlying characteristics of the interaction between buyers and sellers. This naturally led us to analyze how changes in these underlying characteristics affect the equilibrium of the game. Of particular interest is the finding that when trade is intermediated by platforms, buyers and sellers have even more reasons to welcome product differentiation, not only as it does satisfy buyers' taste for variety and reduces competition among sellers, but also because it drives platforms to charge lower access fees. However, buyers and sellers may rank differently markets with different numbers of sellers, and they may do so for opposite reasons than in an environment where trade is not intermediated: in markets where sellers are more numerous, platforms may set higher buyer fees and smaller seller fees, to such an extent that sellers are better off, and buyers worse off, in these markets with stronger competition.

A natural extension of this paper would be to allow for multihoming on the seller or on the buyer side. First efforts in this direction show that it is possible to come up with a fairly simple expression of the platforms' fees at the symmetric equilibrium of the game (thereby, generalizing the results that Belleflamme and Peitz (2010) obtain in the competitive bottlenecks cases). However, when applying the same microfoundations as in this paper, the equilibrium surplus and profit functions for buyers, sellers and platforms 
are complex to a point that comparative statics exercises become hardly tractable. Further research efforts are thus needed.

A less ambitious, yet instructive, route would be to combine the general formulation of Section 2 with other microfoundations of the interaction between the members of the two groups on a platform. Sticking with e-commerce platforms, one could study B2B settings where, presumably, negative within-group effects would also exist among buyers. One could also extend the traditional analysis of other types of platforms by taking withingroup effects into account. For instance, the competition among advertisers on media platforms, or the positive direct network effects among gamers in game console markets could be addressed in this way.

Finally, it would be interesting to endogenize the changes in the degree of competition on the market, by allowing either the entry of sellers, or investments in product differentiation. Yet, this line of research seems more demanding, as it implies substantial extensions of the baseline model of platform competition.

\section{Appendix}

\subsection{Proof of Proposition 1}

Totally differentiating system (3), we have

$$
\left\{\begin{array}{l}
\left(\Delta_{b}^{u}-2 \frac{\tau_{b}}{N_{b}}\right) d n_{b}^{i}+\Delta_{s}^{u} d n_{s}^{i}=d M_{b}^{i}-d M_{b}^{j}, \\
\Delta_{b}^{\pi} d n_{b}^{i}+\left(\Delta_{s}^{\pi}-2 \frac{\tau_{s}}{N_{s}}\right) d n_{s}^{i}=d M_{s}^{i}-d M_{s}^{j} .
\end{array}\right.
$$

Solving the latter system and rearranging terms, we have

$$
\begin{aligned}
d n_{b}^{i} & =\frac{\left(d M_{b}^{i}-d M_{b}^{j}\right)\left(\Delta_{s}^{\pi}-2 \tau_{s} / N_{s}\right)-\left(d M_{s}^{i}-d M_{s}^{j}\right) \Delta_{s}^{u}}{\left(\Delta_{b}^{u}-2 \tau_{b} / N_{b}\right)\left(\Delta_{s}^{\pi}-2 \tau_{s} / N_{s}\right)-\Delta_{b}^{\pi} \Delta_{s}^{u}}, \\
d n_{s}^{i} & =\frac{\left(\Delta_{b}^{u}-2 \tau_{b} / N_{b}\right)\left(d M_{s}^{i}-d M_{s}^{j}\right)-\left(d M_{b}^{i}-d M_{b}^{j}\right) \Delta_{b}^{\pi}}{\left(\Delta_{b}^{u}-2 \tau_{b} / N_{b}\right)\left(\Delta_{s}^{\pi}-2 \tau_{s} / N_{s}\right)-\Delta_{b}^{\pi} \Delta_{s}^{u}} .
\end{aligned}
$$

Using the latter expressions and defining $\phi \equiv\left(\Delta_{b}^{u}-2 \tau_{b} / N_{b}\right)\left(\Delta_{s}^{\pi}-2 \tau_{s} / N_{s}\right)-$ $\Delta_{b}^{\pi} \Delta_{s}^{u}$, we find

$$
\frac{d n_{b}^{i}}{d M_{b}^{i}}=\frac{\Delta_{s}^{\pi}-2 \tau_{s} / N_{s}}{\phi}, \frac{d n_{b}^{i}}{d M_{s}^{i}}=\frac{-\Delta_{s}^{u}}{\phi}, \frac{d n_{s}^{i}}{d M_{b}^{i}}=\frac{-\Delta_{b}^{\pi}}{\phi}, \frac{d n_{s}^{i}}{d M_{s}^{i}}=\frac{\Delta_{b}^{u}-2 \tau_{b} / N_{b}}{\phi} .
$$


We can now turn to the first stage of the game at which platforms set prices (for given sellers' investment levels). Assuming that the intermediary's cost per buyer is $C_{b}$ and per seller is $C_{s}$, we can write platform $i$ 's profit as

$$
\Pi^{i}=\left(M_{b}^{i}-C_{b}\right) n_{b}^{i}\left(M_{b}^{i}, M_{s}^{i} ; M_{b}^{j}, M_{s}^{j}\right)+\left(M_{s}^{i}-C_{s}\right) n_{s}^{i}\left(M_{b}^{i}, M_{s}^{i} ; M_{b}^{j}, M_{s}^{j}\right) .
$$

The two intermediaries simultaneously choose membership fees on both sides of the market. Considering platform $i$, the first-order conditions of profit maximization are:

$$
\begin{aligned}
n_{b}^{i}+\frac{\partial n_{b}^{i}}{\partial M_{b}^{i}}\left(M_{b}^{i}-C_{b}\right)+\frac{\partial n_{s}^{i}}{\partial M_{b}^{i}}\left(M_{s}^{i}-C_{s}\right)=0, \\
\frac{\partial n_{b}^{i}}{\partial M_{s}^{i}}\left(M_{b}^{i}-C_{b}\right)+n_{s}^{i}+\frac{\partial n_{s}^{i}}{\partial M_{s}^{i}}\left(M_{s}^{i}-C_{s}\right)=0 .
\end{aligned}
$$

We focus now on a symmetric equilibrium: $M_{b}^{1}=M_{b}^{2}=M_{b}$ and $M_{s}^{1}=$ $M_{s}^{2}=M_{s}$, which implies that $n_{b}^{i}=n_{b}^{j}=N_{b} / 2$ and $n_{s}^{i}=n_{s}^{j}=N_{s} / 2$. Using this along with expressions (15), we can rewrite the previous two conditions as:

$$
\left\{\begin{array}{c}
\left(\Delta_{s}^{\pi}-2 \frac{\tau_{s}}{N_{s}}\right)\left(M_{b}-C_{b}\right)-\Delta_{b}^{\pi}\left(M_{s}-C_{s}\right)=-\frac{1}{2} \phi N_{b}, \\
-\Delta_{s}^{u}\left(M_{b}-C_{b}\right)+\left(\Delta_{b}^{u}-2 \frac{\tau_{b}}{N_{b}}\right)\left(M_{s}-C_{s}\right)=-\frac{1}{2} \phi N_{s} .
\end{array}\right.
$$

Solving the latter system of equations, we find the Nash equilibrium membership fees of the two platforms as

$$
\begin{aligned}
& M_{b}^{*}=C_{b}+\tau_{b}-\frac{N_{b}}{2} \Delta_{b}^{u}-\frac{N_{s}}{2} \Delta_{b}^{\pi}, \\
& M_{s}^{*}=C_{s}+\tau_{s}-\frac{N_{b}}{2} \Delta_{s}^{u}-\frac{N_{s}}{2} \Delta_{s}^{\pi} .
\end{aligned}
$$

\subsection{Effects of stronger competition on sellers' net surplus}

Using expression (13), we compute the following derivatives.

$\frac{\partial v_{s}^{*}}{\partial \gamma}=2 N_{b} \frac{\left(N_{s}-2\right)^{2} \gamma^{2}+8\left(N_{s}-2\right) \gamma-8\left(3 N_{s}-2\right)}{\left(4+\gamma\left(N_{s}-2\right)\right)^{4}}>0 \Leftrightarrow \gamma>\frac{2 \sqrt{6 N_{s}}-4}{N_{s}-2} ;$

the latter inequality cannot be satisfied if the threshold is larger than unity, which is equivalent to $N_{s} \leq 19.798$.

$$
\begin{aligned}
\frac{\partial v_{s}^{*}}{\partial N_{s}} & =4 \gamma N_{b} \frac{\gamma^{2} N_{s}-2(2-\gamma)(3-\gamma)}{\left(4+\gamma\left(N_{s}-2\right)\right)^{4}}>0 \Leftrightarrow N_{s}>\frac{2(2-\gamma)(3-\gamma)}{\gamma^{2}}, \\
\frac{\partial v_{s}^{*}}{\partial N_{b}} & =2 \frac{2(2-\gamma)^{2}-\gamma^{2} N_{s}}{\left(4+\gamma\left(N_{s}-2\right)\right)^{3}}>0 \Leftrightarrow N_{s}<\frac{2(2-\gamma)^{2}}{\gamma^{2}} .
\end{aligned}
$$




\subsection{Effects of product substitutability on platforms' profits}

Using expression (14), we compute:

$$
\frac{\partial \Pi^{*}}{\partial \gamma}=N_{b} N_{s} \frac{-\left(N_{s}-2\right)^{2} \gamma^{2}+2\left(N_{s}-2\right)\left(N_{s}-6\right) \gamma+32\left(N_{s}-1\right)}{\left(4+\gamma\left(N_{s}-2\right)\right)^{4}},
$$

which is positive provided that

$$
\gamma<\frac{N_{s}-6+\sqrt{20 N_{s}+N_{s}^{2}+4}}{N_{s}-2} .
$$

The latter threshold is larger than unity if $20 N_{s}+N_{s}^{2}-12>0$, which is satisfied for any $N_{s} \geq 2$. It follows that $\partial \Pi^{*} / \partial \gamma>0$ for the whole admissible range of parameters.

\section{References}

[1] Armstrong, M. (2006), Competition in Two-Sided Markets, Rand Journal of Economics 37, 668-691.

[2] Armstrong, M. and J. Wright (2007), Two-sided Markets, Competitive Bottlenecks and Exclusive Contracts, Economic Theory 32, 353-380.

[3] Bardey, D., Cremer, H., Lozachmeur, J.M. (2014). Competition in Two-Sided Markets with Common Network Externalities. Review of Industrial Organization 44, $327-359$.

[4] Belleflamme, P. and Jacqmin, J. (2016). An Economic Appraisal of MOOC Platforms: Business Models and Impacts on Higher Education. CES-IFO Economic Studies $62,148-169$.

[5] Belleflamme, P., Omrani, N. and Peitz, M. (2015). The Economics of Crowdfunding Platforms. Information Economics and Policy 33, 11-28.

[6] Belleflamme, P. and Peitz, M. (2010). Platform Competition and Seller Investment Incentives. European Economic Review 54, 1059-1076.

[7] Belleflamme, P. and Toulemonde, E. (2009). Negative Intra-Group Externalities in Two-Sided Markets. International Economic Review 50, 245-272.

[8] Caillaud, B., Jullien, B. (2003). Chicken and Egg: Competition among Intermediation Service Providers. Rand Journal of Economics 34, 521-552. 
[9] Ellison, G. and Fudenberg, D. (2003). Knife-Edge or Plateau: When Do Market Models Tip? Quarterly Journal of Economics 118, 1249-1278.

[10] Ellison, G., Fudenberg, D., and Möbius, M. (2004). Competing Auctions. Journal of the European Economic Association 2, 30-66.

[11] Evans, D. (2003), Some Empirical Aspects of Multi-sided Platform Industries, Review of Network Economics 2, 191-209.

[12] Galeotti, A. and Moraga-González, J.L. (2009). Platform Intermediation in a Market for Differentiated Products, European Economic Review 53, 417-428.

[13] Hagiu, A. (2006). Price and Commitment by Two-Sided Platforms. Rand Journal of Economics 37, 720-737.

[14] Hagiu, A. (2009). Two-Sided Platforms: Product Variety and Pricing Structures. Journal of Economics and Management Strategy 18,1011-1043.

[15] Karle, H., Peitz, M., and Reisinger, M. (2015). Segmentation versus Agglomeration: Competition between Platforms with Competitive Sellers. Mimeo.

[16] Lin, M., Li, S., and Whinston, A.B. (2011). Innovation and Price Competition in a Two-Sided Market. Journal of Management Information Systems 28, 171-202.

[17] Martin, S. (2009). Microfoundations for the Linear Demand Product Differentiation Model, with Applications. Mimeo.

[18] Nocke, V., Peitz, M., and Stahl, K. (2007). Platform Ownership, Journal of the European Economic Association 5, 1130-1160.

[19] Reisinger, M. (2014). Two-Part Tariff Competition between Two-Sided Platforms. European Economic Review 68, 168-180.

[20] Rochet, J.-C. and Tirole, J. (2003). Platform Competition in Two-Sided Markets. Journal of the European Economic Association 1, 990-1024.

[21] Rochet, J.-C. and Tirole, J. (2006). Two-Sided Markets: A Progress Report. Rand Journal of Economics 37, 645-667.

[22] Shubik, M. and Levitan, R. (1980). Market Structure and Behavior. Cambridge, Massachusetts: Harvard University Press.

[23] Singh, N. and Vives, X. (1984). Price and Quantity Competition in a Differentiated Duopoly. Rand Journal of Economics 15, 546-554. 
[24] Slee, T. (2016). Airbnb Is Facing an Existential Expansion Problem. Harvard Business Review. Online July 11, 2016 (https://hbr.org/2016/07/airbnb-is-facing-anexistential-expansion-problem).

[25] Tavalaei, M.M. and Santalo, J. (2015). The Impact of Within Platform Competition in Two-Sided Business Models. Academy of Management Annual Meeting Proceedings.

[26] Weyl, E.G. (2010). A Price Theory of Multi-Sided Platforms. American Economic Review 100, 1642-1672.

[27] Weyl, E.G. and A. White (2016). Insulated Platform Competition. Mimeo. Available at SSRN: http://ssrn.com/abstract=1694317. 